\title{
Pattern of dermatoses in two groups of admitted psychiatric patients: a cross-sectional study from a tertiary care hospital in Kashmir
}

\author{
Tasleem Arif ${ }^{1 凶}$, Iffat Hassan ${ }^{1}$, Mushtaq A. Margoob², Parvaiz Anwar ${ }^{1}$, Sheikh Shoib², Syed Akeel²
}

\begin{abstract}
Background: Various specific and non-specific dermatological manifestations can be found in patients with psychiatric ailments. Most studies in this regard have been conducted on an outpatient basis and not much work has been done on patients admitted with psychiatric diseases.

Methods: This cross-sectional hospital-based study involved two groups of admitted psychiatric patients over a period of 1 year, involving 100 patients in each group. In the family ward group patients were admitted with accompanying family members, whereas in the closed ward group patients were kept under custodial care.

Results: In the family ward setting, eczema was the most common finding, observed in 29 patients, followed by atrophic scarring in 28 patients, erythema ab igne in 25 patients, and bacterial infections in five patients. Various forms of nail changes were seen in 18 patients. In the closed ward group, most common dermatological involvement was parasitic infestation, seen in 56 patients, followed by generalized pruritus in 53 patients and atrophic scarring in 52 patients. Thirty-eight patients had nail changes.

Conclusion: Skin manifestations are more common in chronic neglected psychiatric patients under custodial care. The authors stress upon the importance of familial care provided to psychiatric patients living in custodial settings.
\end{abstract}

Keywords: atrophic scarring, dermatological manifestations, eczema, parasitic infestations, psychiatric patients

Received: 14 April 2017 | Returned for modification: 29 June 2017 | Accepted: 12 October 2017

\section{Introduction}

Psychodermatology involves the interaction between the mind and the body. Dermatology addresses the external "visible" disease, whereas psychiatry deals with the internal "invisible" disease (1). Psychological factors may play a role in many chronic skin diseases such as prurigo, acne, alopecia, psoriasis, and eczema $(2,3)$. Cutaneous manifestations are common in patients with primary psychiatric illness (4). Conversely, more than $75 \%$ of patients with dermatological diseases have associated psychological problems (5). Data regarding the pattern of skin diseases in psychiatric patients are scarce. Most studies published on this subject are mainly concerned with screening outpatient psychiatric subjects and some chronic psychiatric patients treated in custodial care setups. To the best of our knowledge, not much has been reported regarding the spectrum of skin manifestations in psychiatric patients admitted in various settings, such as those having family members with them as caretakers, and those living in closed wards under custodial care and neglected by the family. This inspired us to study the pattern and spectrum of cutaneous manifestations in these two setups.

\section{Methods}

This study was carried out by Department of Dermatology and Department of Psychiatry at Government Medical College Srinagar, a tertiary care hospital of Kashmir valley of India. It was a cross-sectional study that involved only admitted patients with a primary psychiatric disorder at this hospital over the period of 1 year, from October 2012 to September 2013. The patients for the study were categorized into two groups as follows:
Family ward: The first group comprised 100 psychiatric patients that were admitted to family wards with accompanying family members. These patients usually stay for a short duration of a few days or weeks (less than 1 month), usually admitted for exacerbation of their primary psychiatric disorder, and are well taken care of by their accompanying family members or relatives during their hospital stay. Once their symptoms improve, they are soon discharged.

Inclusion criteria: 1) Diagnosis of psychiatric illness, 2) Admission to the hospital for less than 1 month, 3) Patient accompanied by one or more caretakers, 4) Residing in open wards with accompanying caretaker(s) free to move out.

Closed Ward: The second group comprised 100 patients mostly suffering from longstanding schizophrenia that have been in the hospital for many months to years, mostly neglected by their families. They were kept in closed wards (custodial care) under security because many of them have lost any perspective on their disease. This group of patients was at some disadvantages such as lack of personal care, self-inflicted injuries, negligence by their families, and lack of interest from healthcare staff because some of them have become so aggressive as to pose a threat to other people; the long duration of disease was reflected in their skin manifestations.

Inclusion criteria: 1) Diagnosis of psychiatric illness, 2) Admission to the hospital for more than 1 month (usually months to years), 3) Patients not accompanied by caretakers, 4) Residing under custodial settings (under security arrangements).

Exclusion criteria (common for both groups): 1) Age less than 12 years, 2) Pregnancy or history of pregnancy in the last 6 months, 3) Patients whose dermatological condition was diagnosed before the onset of primary psychiatric illness. 
Diagnoses of psychiatric disorders: The diagnosis of psychiatric disorder was made by a consultant psychiatrist according to the criteria in the Diagnostic and Statistical Manual of Mental Disorders, fourth edition (DSM-IV) (6). Informed consent was obtained from each patient, and those considered incapable of consenting participated with the consent of their closest family member or custodian. The study was approved by the ethical committee of Government Medical College Srinagar.

Data were collected regarding demographic variables such as age, sex, marital status, occupation, and psychiatric diagnosis. A detailed dermatological examination was carried out by the same dermatologist for all patients. Relevant investigations such as scraping for fungus and biopsy for histopathological examination were made when required. Consent to publish the individual patient data, including images, was provided by the patients or by their guardian or custodian.

At the end of the study, the data were compiled and analyzed statistically using the computer program SPSS version 16. Tests including chi-squared and Fisher's exact test were applied at appropriate sites, and $p<0.05$ was considered significant.

\section{Results}

Family ward setting: There were 100 patients in this study group, 72 males and 28 females, with a male-to-female ratio of 2.57:1. The patient age ranged from 12 to 76 years with an average of 32.6 years. The majority of patients (36) were in their third decade (Fig. 1). Sixty-two patients were unmarried (including divorced) and 38 were married, yielding an unmarried-to-married ratio of 1.63:1. Sixty-four patients were from rural backgrounds and 36 from ur-

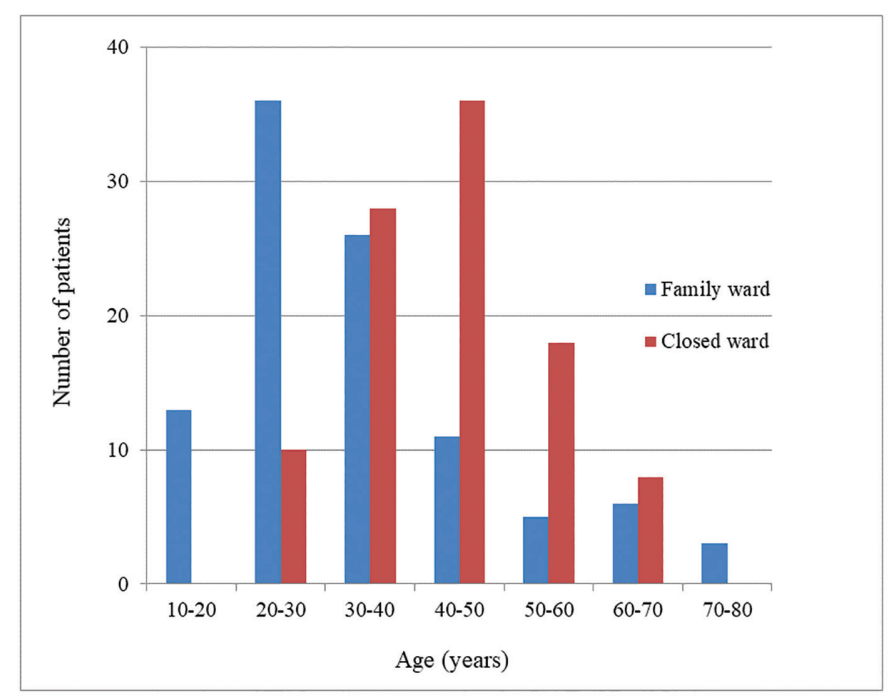

Figure 1 | Age distribution in the two patient groups.

Table 1 | Psychiatric diagnoses in the two patient groups.

\begin{tabular}{|c|c|c|c|c|c|c|c|}
\hline Diagnosis & NF & NC & $p$-value & Diagnosis & NF & NC & $p$-value \\
\hline BPAD & 53 & 8 & $<0.001$ & Dementia with Parkinsonism & 1 & 0 & 1.000 \\
\hline Mania & 31 & & & & & & \\
\hline Depression & 20 & & & & & & \\
\hline Conversion & 2 & & & & & & \\
\hline Schizophrenia & 17 & 86 & $<0.001$ & ADHD & 1 & 0 & 1.000 \\
\hline Substance abuse & 16 & 12 & 0.541 & Anxiety & 2 & 0 & 0.498 \\
\hline MDD & 7 & 0 & 0.021 & Personality disorder & 1 & 0 & 1.000 \\
\hline OCD & 5 & 0 & 0.059 & Dissociative disorder & 1 & 0 & 1.000 \\
\hline Conversion disorder & 2 & 0 & 0.498 & Delusional disorder & 1 & 0 & 1.000 \\
\hline Somatization disorder & 1 & 0 & 1.000 & Seizure disorder & 1 & 0 & 1.000 \\
\hline Conduct disorder & 1 & 0 & 1.000 & Schizoaffective disorder & 0 & 2 & 0.498 \\
\hline
\end{tabular}

ban areas, with a rural-to-urban ratio of 1.77:1. The occupation of the patients showed diversity (Fig. 2), with students predominating (24).

Bipolar affective disorder (BPAD) topped the list among psychiatric diagnoses, comprising 53 patients (Table 1).

Among the skin manifestations, eczema was present in 29 patients, followed by atrophic scarring in 28 (Fig. 3) and erythema ab igne in 25 patients. The various skin manifestations are summarized in Table 2. Nail changes (including both fingernails and toenails) were seen in 18 patients (Table 3), of which longitudinal ridging was the most common finding, seen in 10 cases.

Closed ward setting: In this group, 100 patients were studied. The average age was 41 years, with a range of 21 to 65 years. Males comprised $86 \%$ and females constituted $14 \%$ of cases, with a male-to-female ratio of 6.14:1. Sixty-six patients were unmarried and 34 patients were married, yielding an unmarried-to-married ratio of 1.94:1. Seventy patients belonged to rural areas and 30 patients were from urban areas, with a rural-to-urban ratio of 2.33:1. The majority of patients in this study group ( 36 patients) were in their fifth decade (Fig. 1). Regarding occupation (Fig. 2), 22 patients were farmers, followed by laborers (12 patients).

Among the psychiatric diagnoses (Table 1), schizophrenia was the most common diagnosis, seen in 86 cases. Of all the patients, eight had criminal records.

Parasitic infestation was the most common skin manifestation, seen in 56 patients, of which 38 had pediculosis (Fig. 4) and 28 had scabies. The various cutaneous manifestations are summarized in Table 2 (Figs. $5^{-8}$ ).

Nail changes (including both fingernails and toenails) were seen in 38 patients, of which ridging was the most common finding, seen in 20 patients. The various nail changes are mentioned in Table 3 (Fig. 9). It should be mentioned here that some patients had more than one type of psychiatric disorder. Similarly, many patients also had more than one type of skin manifestation.

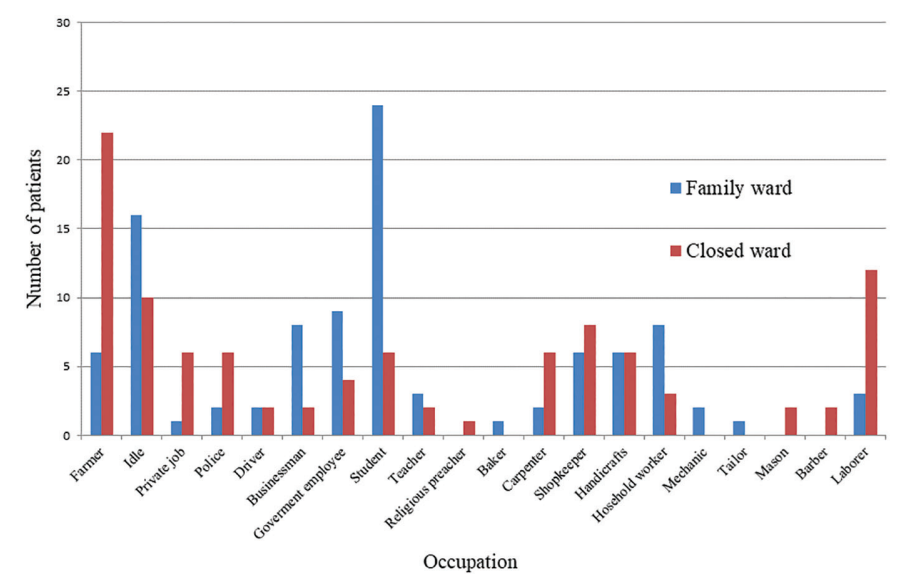

Figure 2 | Occupation of the two patient groups.

$\mathrm{D}=$ bipolar affective disorder $\mathrm{MDD}=$ major depressive disorder; $\mathrm{OCD}=$ obses
sive compulsive disorder, $A D H D=$ attention deficit hyperkinetic disorder. 


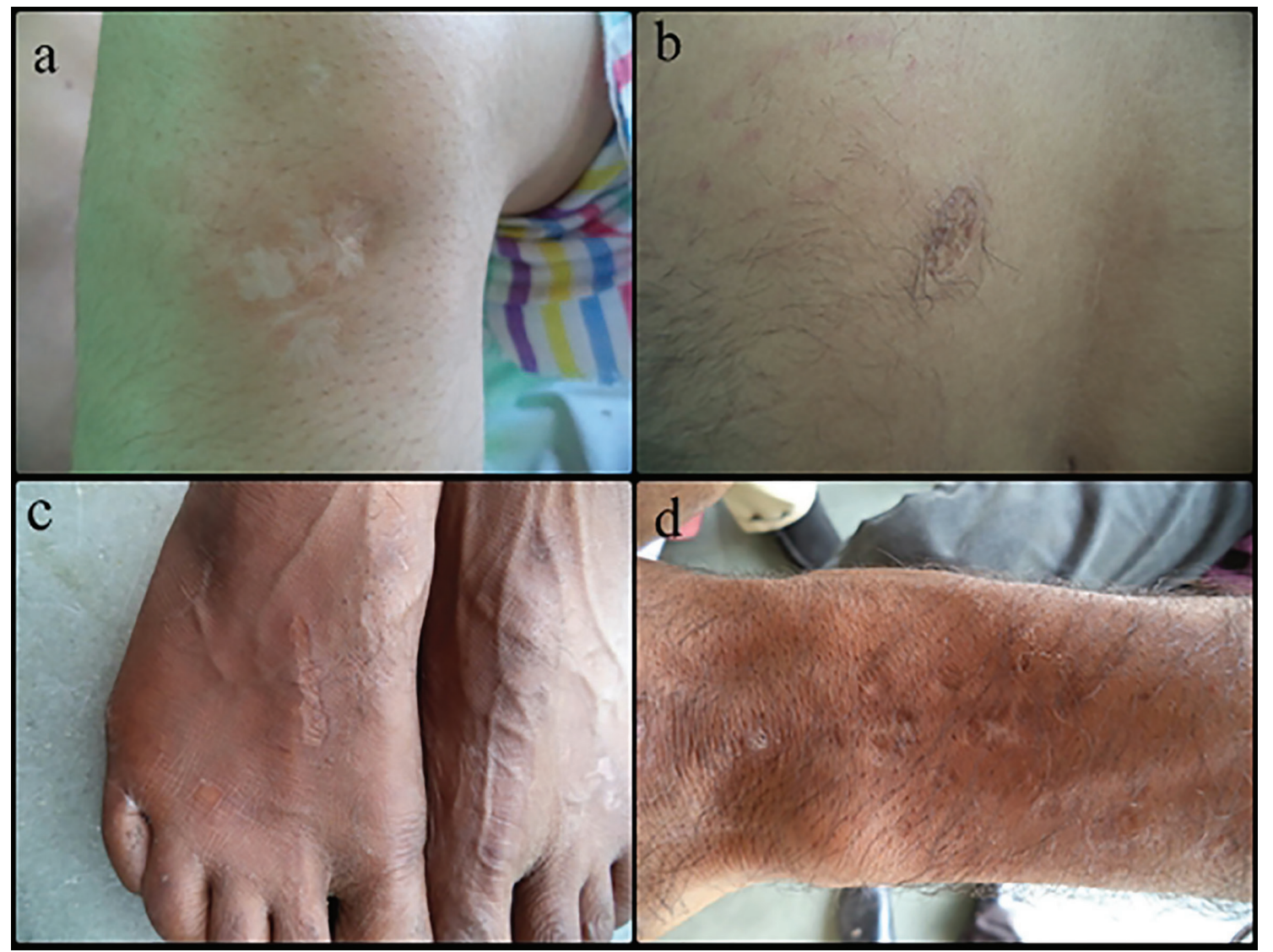

Figure $3 \mid$ Atrophic scarring on the leg (a), back (b), dorsal aspects of feet (c), and forearm (d).

Table 2 | Various cutaneous manifestations in the two groups of patients.

\begin{tabular}{|c|c|c|c|c|c|c|c|}
\hline Skin manifestation & $\mathrm{NF}$ & NC & $p$-value & Skin manifestation & NF & NC & $p$-value \\
\hline Parasitic infestations & 2 & 56 & & & & & \\
\hline Pediculosis & & 38 & $<0.001$ & Hyperhidrosis & 13 & 6 & 0.091 \\
\hline Scabies & & 28 & & & & & \\
\hline Generalized pruritus & 2 & 53 & $<0.001$ & Delusional parasitosis & 4 & 6 & 0.745 \\
\hline Atrophic scarring & 28 & 52 & $<0.001$ & Prurigo & 0 & 6 & 0.038 \\
\hline Nail changes & 18 & 38 & 0.002 & Acne excoriee & 10 & 4 & 0.166 \\
\hline Eczema & 29 & 26 & 0.635 & LSC & 2 & 4 & 0.679 \\
\hline Fungal infections & 2 & 18 & $<0.001$ & Anetoderma & 0 & 4 & 0.121 \\
\hline Xerosis & 5 & 18 & 0.004 & Scarring alopecia, scalp (traumatic) & 0 & 4 & 0.123 \\
\hline Hypo- and hyperpigmentation & 0 & 12 & 0.001 & KP & 3 & 2 & 1.000 \\
\hline Neurotic excoriation & 1 & 10 & 0.013 & Trichotillomania & 3 & 2 & 1.000 \\
\hline Bacterial infection & 5 & 8 & 0.566 & Punctate atrophoderma & 0 & 2 & 0.498 \\
\hline Seborrheic dermatitis & 14 & 8 & 0.175 & Erythema ab igne & 25 & 2 & $<0.001$ \\
\hline Macular amyloidosis & 0 & 8 & 0.012 & Burns & 7 & 0 & 0.021 \\
\hline Pellagra & 1 & 0 & 1.000 & Psoriasis & 2 & 0 & 0.498 \\
\hline Alopecia areata & 1 & 0 & 1.000 & Ichthyosis & 2 & 0 & 0.498 \\
\hline Atopic dermatitis & 1 & 0 & 1.000 & Hirsutism & 4 & 0 & 0.121 \\
\hline Maculopapular rash & 1 & 0 & 1.000 & Terraforme dermatitis & 2 & 0 & 0.498 \\
\hline Suicidal cuts & 2 & 0 & 0.498 & IV injection marks & 1 & 0 & 1.000 \\
\hline Chronic urticaria & 1 & 0 & 1.000 & Callosities & 2 & 0 & 0.498 \\
\hline Black hairy tongue & 1 & 0 & 1.000 & & & & \\
\hline
\end{tabular}

$\mathrm{NF}=$ number of patients in family ward, $\mathrm{NC}=$ number of patients in closed ward, $\mathrm{LSC}=$ lichen simplex chronicus, $\mathrm{KP}=$ keratosis pilaris.

Table 3 | Nail changes in the two groups of patients.

\begin{tabular}{|c|c|c|c|c|c|c|c|}
\hline Nail change & NF & $\mathrm{NC}$ & $p$-value & Nail change & $\mathrm{NF}$ & NC & $p$-value \\
\hline Longitudinal ridging & 10 & 20 & 0.047 & Thickening of nail plate & 2 & 6 & 0.279 \\
\hline Beau's lines & 3 & 8 & 0.215 & Dystrophic nails & 2 & 10 & 0.037 \\
\hline Longitudinal melanonychia & 3 & 6 & 0.495 & Traumatic nails & 3 & 8 & 0.215 \\
\hline Onycholysis, focal & 2 & 2 & 1.000 & Punctuate leuconychia & 2 & 14 & 0.004 \\
\hline Pitting & 3 & 0 & 0.246 & Paronychia & 3 & 8 & 0.215 \\
\hline Pigmentation, focal & 3 & 5 & 0.721 & Median canalicular dystrophy of Heller & 0 & 6 & 0.038 \\
\hline Complete pigmentation of nail plate & 0 & 4 & 0.121 & Petechial hemorrhage & 0 & 8 & 0.012 \\
\hline Clubbing & 0 & 1 & 1.000 & & & & \\
\hline
\end{tabular}

Clubbing

Total nail changes in closed ward patients $=38 \%(n=38), p=0.002$

$\mathrm{NF}=$ number of patients in family ward, $\mathrm{NC}=$ number of patients in closed ward, $\mathrm{LSC}=$ lichen simplex chronicus, $\mathrm{KP}=$ keratosis pilaris. 


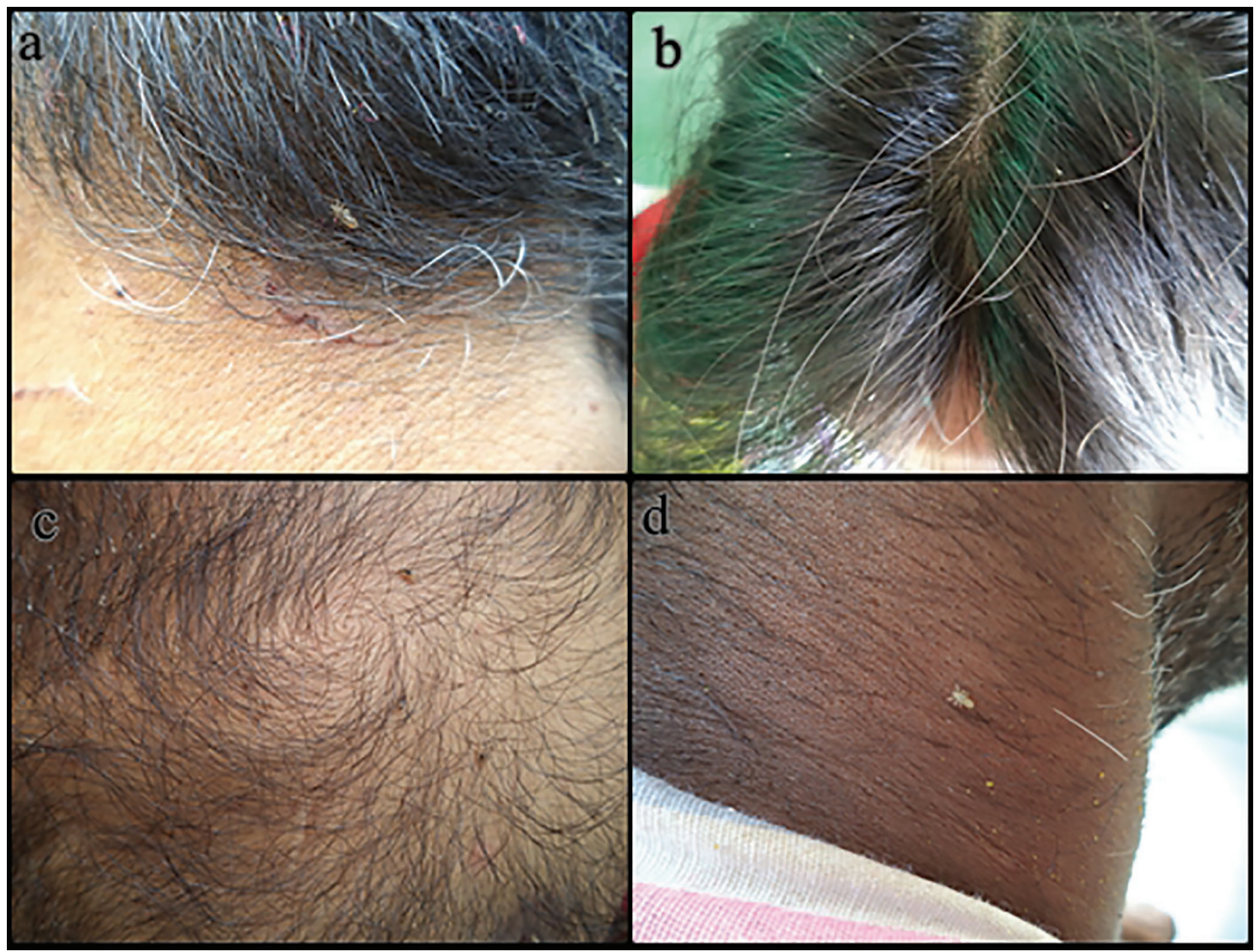

Figure 4 | Pediculosis capitis (a, b) and pediculosis corporis (c, d) in chronic schizophrenic patients.

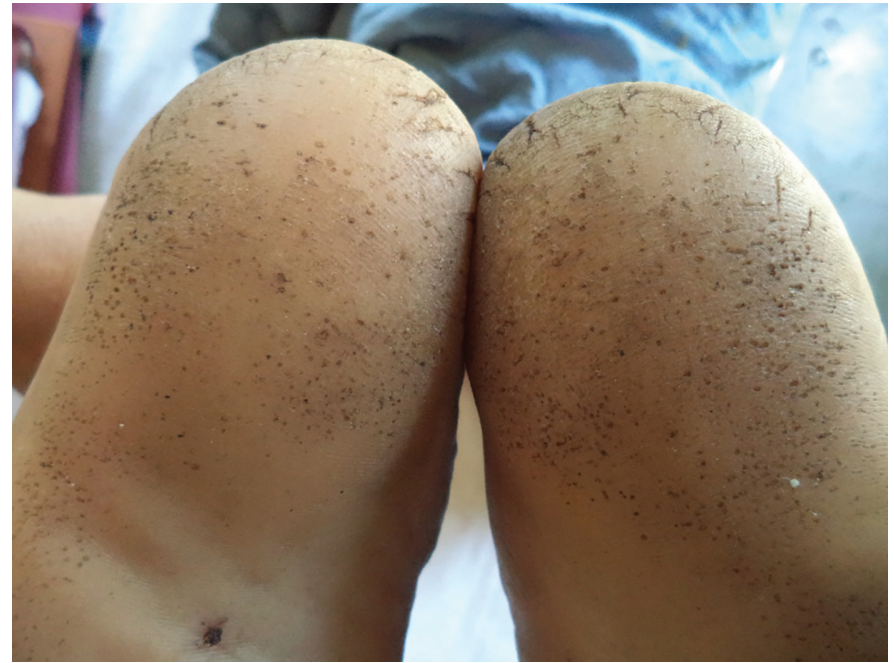

Figure 5 | Pitted keratolysis on the soles.

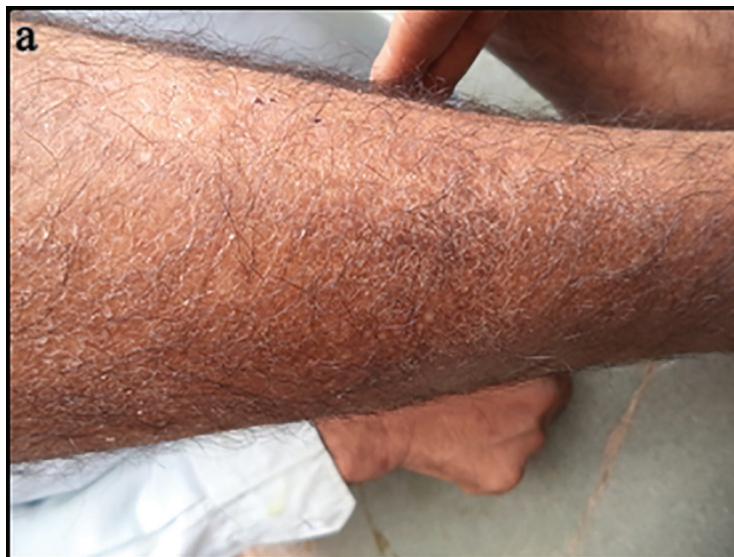

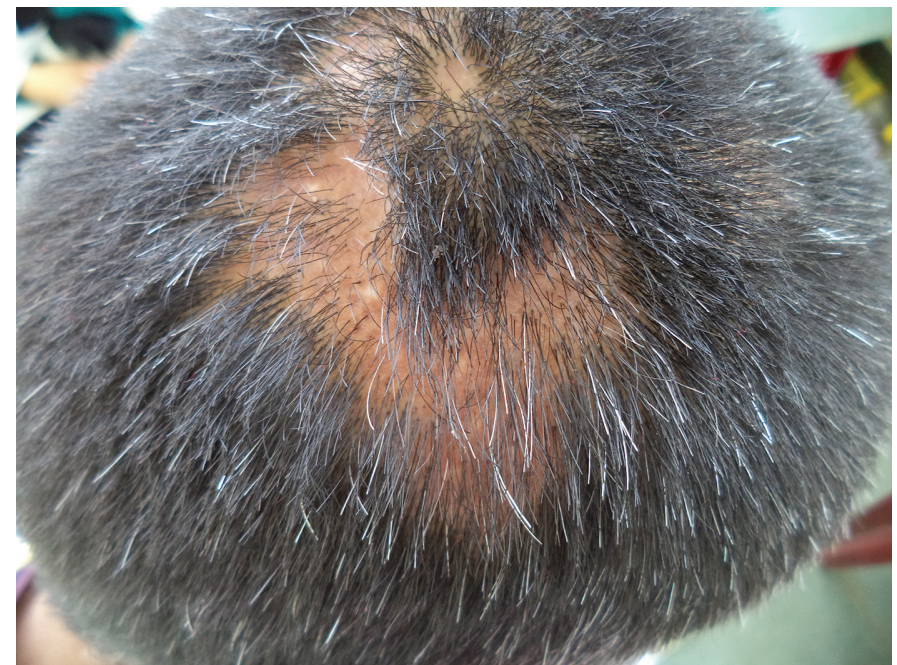

Figure 7 | Scarring alopecia on the scalp.

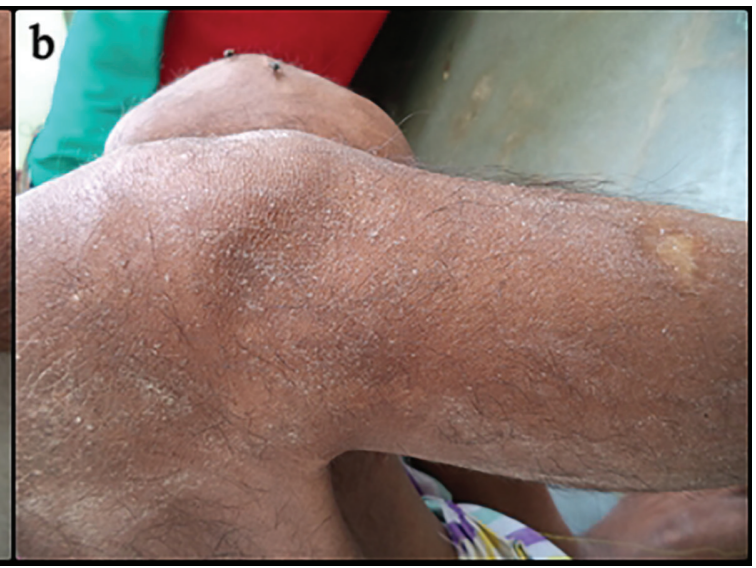

Figure $6 \mid$ Xerosis on the arm (a) and leg (b). 


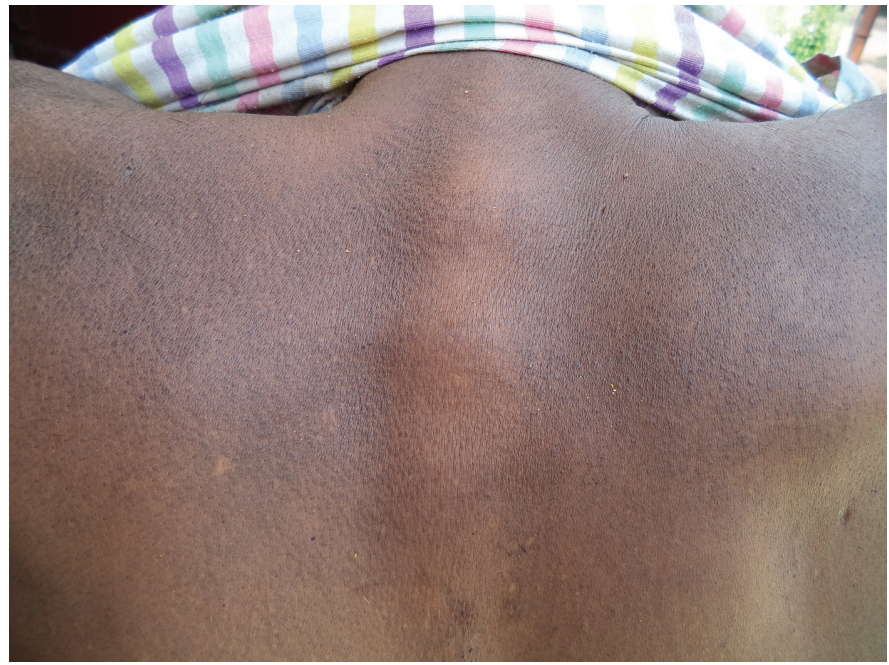

Figure 8 | Macular amyloidosis on the shoulders and upper back.

\section{Discussion}

This study was carried out on two groups of admitted patients with psychiatric diseases. In both groups there was a male predominance, which was more marked in the closed ward setup (male-to-female ratio of 6.14:1) than in family ward settings (maleto-female ratio of 2.57:1), which is similar to the previous study by Mookhoek et al. (7). They studied two groups (short-stay and longstay patients) and showed a predominance of males in both study groups, with male-to-female numbers at 87:60 and 66:42, respectively (7). However, a study by Kuruvilla et al. showed a female predominance in 300 patients with primary psychiatric disorders studied for associated skin manifestations (8). However, the male predominance in our study can be explained in several ways. First, the studies mentioned above involved both outpatient department cases as well as admitted patients, whereas in our case there were only admitted patients. Second, family members show more reluctance to admit females to a psychiatric hospital. Third, many patients in our study group were drug abusers and there has been a very high predominance of males among drug abusers, which is confirmed by the study by Margoob et al. (9).

The majority of cases were in their third to fifth decades, consistent with previous studies (8). Whereas the age group of the third decade dominated the family group, the closed ward setup showed a preponderance of patients in their fifth decade. Regarding the occupation of patients in both groups, diversity was noticed and various occupations involved. A conspicuous finding was that the majority of patients in the family ward setup were students, comprising 24 cases. This can be explained by the fact that Kashmir is a chronic conflict zone and has been in a state of turmoil for many years, and this has increased psychiatric morbidity among people, with resulting effects on education (10). Second, 16 patients in the family ward were drug abusers, and this is seen more among young students. A study by Rather et al. on the socio-demographic and clinical profile of substance abusers attending a regional drug addiction treatment center in the Kashmir Valley revealed that $78.8 \%$ of drug abusers were primary and high school students (11).

In the family setup, BPAD affected 53 patients, followed by schizophrenia (17 patients) and substance abuse (16 patients). In contrast, the closed setup was dominated by schizophrenia, found in 86 patients, followed by substance abuse (12 patients) and BPAD (eight patients). Mookhoek et al. (7) also found psychosis as a major diagnosis in long-stay patients (77\%), but among

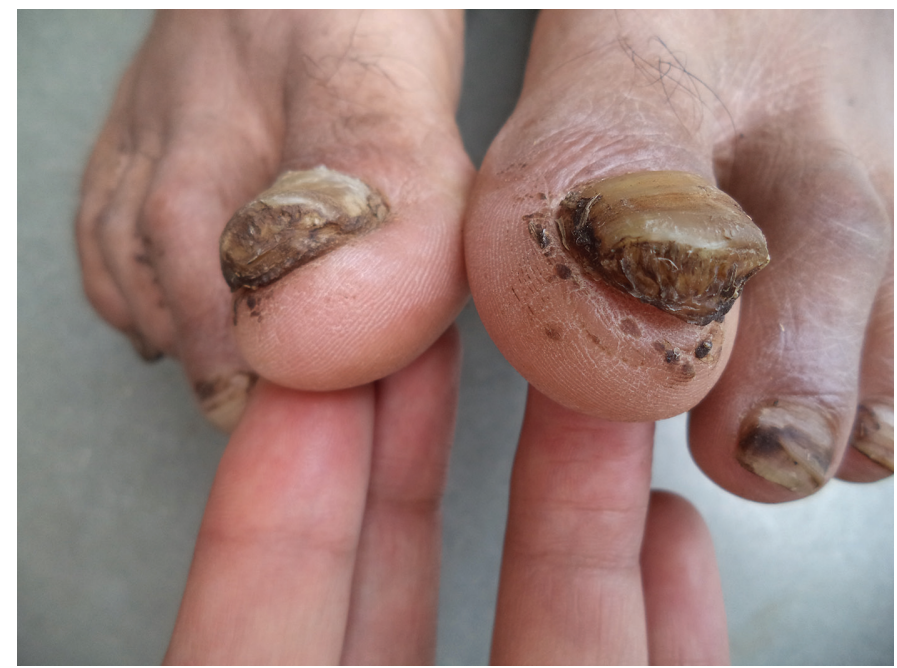

Figure 9 | Abnormally thickened nail plates.

short-stay patients, BPAD ranked number two in diagnosis (23\%) after psychosis (39\%), which differed from our study. This difference can be attributed to the different inclusion criteria as in their study: short-stay patients referred to admission time less than 1 year, whereas in this study the family ward setting referred to a stay of less than 1 month in the hospital.

Regarding the spectrum of cutaneous manifestations of family setup, noninfective skin disease was the major pattern, found in 91 patients. Eczema was the most common skin disorder, seen in 29 patients, which is comparable to previous studies (7). On the other hand, parasitic infections and infestations were the commonest skin manifestations, found in 56 patients in the closed ward setup in contrast to the family ward setup (two patients), with a statistically significant difference $(p<0.001)$. This high prevalence of parasitic infections and infestations is consistent with previous studies $(7,8)$. Parasitic infestations (scabies, pediculosis) in patients in closed wards can result from transmission of these infections among patients. Appropriate hygienic measures such as separate towels and sheets, regular bathing, and prevention of overcrowding can prevent such transmission.

One of the skin manifestations prevalent in both groups of patients was atrophic scarring, which was more common in closed ward patients (52 patients) than in the family ward ( 28 patients), with a statistically significant difference $(p<0.001)$. Various reasons appeared to be responsible for high prevalence of atrophic scarring in our study, including sedation with drugs and consequent trauma and scarring, self-inflicted trauma, quarrels due to violent behavior, and burns. Some studies involving skin manifestations in psychiatry patients used a pre-defined classification of psycho-cutaneous disorders, in which the parameter "atrophic scarring” was missing (8). Second, our study groups had a good percentage of substance abusers, which are associated with selfinflicted trauma and consequent atrophic scarring (12). Williams demonstrated that $15 \%$ had scars from self-inflicted injury in a psychiatric population (13). This may also be a reflection of lack of personal and family care, self-inflicted injuries leading to scarring, quarrels between violent patients culminating in injuries and scarring, burns due to carelessness, and less attention provided by healthcare staff towards aggressive and violent patients, so that scarring is almost twice as frequent in custodial patients compared to family ward patients.

Another unusual finding in our study was the presence of erythema ab igne, seen in 25 patients in the family ward group and only two of the closed ward patients, with a statistically signifi- 
cant difference $(p<0.001)$. The prolonged use of a kangri leads to erythema ab igne, a brownish-blue reticulate hyperpigmentation with erythema that may transform into cancer (14). A kangri is a traditional earthen fire pot used by Kashmiris, kept close to body between the abdomen and thighs to keep the body warm during winter months (15). It can also be used for warming acral parts such as the hands and feet. The low percentage of erythema $a b$ igne in the closed group (two patients only) in comparison to the family group can be explained by their relative lack of exposure to extremes of cold because they have been in closed wards for a long time and have fixed heating arrangements in the wards, so that they do not need any personal heating devices like a kangri.

Generalized pruritus was seen in 53 patients in the closed group and in only two patients in the family ward patients $(p<$ o.001), which can be explained on the basis of untreated scabies and pediculosis, along with a higher number of xerotic patients in the closed group patients. The higher prevalence of acne excoriee in the family group (10 patients) than in the closed group (four patients) may be because the family ward group comprised younger patients than the closed ward group.

Macular amyloidosis due to amyloid deposition in the skin is characterized by clusters of small pigmented macules, which may coalesce to produce macular hyperpigmented areas commonly seen on the back and chest, usually as a result of chronic friction (16). In our study, eight patients from the closed group had macular amyloidosis involving the shoulders, lower back, and chest, and none from family ward had it, which can be attributed to the chronicity of pruritus and consequent friction.

Hyperhidrosis is excessive sweating usually due to overactivity of cholinergic innervations of the sweat glands. It can be primary (idiopathic) or secondary to some other conditions such as diabetes mellitus, thyrotoxicosis, anxiety, menopause, peripheral neuropathies, Parkinsonism, and drugs such as selective serotonin reuptake inhibitors (SSRI), opioids, and tricyclic antidepressants (17). In this study, 13 patients in family group had hyperhidrosis, eight of them were receiving SSRI (three fluvoxamine, two escitalopram, two mirtazapine, and one fluoxetine), one was suffering from Parkinsonism, one hyperthyroidism, one diabetes mellitus, one associated with pitted keratolysis of soles, and in the other the cause was not known. However, in the closed group, hyperhidrosis was seen in six patients, among whom four had associated pitted keratolysis of the feet and in another two the reason was not known. The higher prevalence in the family group can be attributed to concomitant use of SSRI for depression.

In delusional parasitosis, patients have the false belief that their bodies are infested by organisms and such patients often present with bits of excoriated skin, insect parts, or debris to sup- port their argument (1). It can be primary or secondary to organic diseases such as cerebrovascular disease, Parkinsonism, multiple sclerosis, diabetes, hypothyroidism, hyperthyroidism, pellagra, malignancy, dementia, and substance abuse, such as cannabis abuse (18). In our study, four patients in the family ward had delusional parasitosis, among whom one had Parkinsonism with dementia, one had pellagra, and two were young students with cannabis substance abuse. In the closed setup, six patients had delusional parasitosis and probably all of them developed it following real infestation with scabies, which is consistent with a previous study by Lyell (19).

Trichotillomania is an impulse-control disorder with a craving for pulling out one's hair and is usually seen in depression, anxiety disorder, and obsessive-compulsive disorder (OCD) (20). In our study, it was seen in three patients in the family group (suffering from depression, OCD, and anxiety) and two patients from the closed group, both associated with depression.

Nail changes (including both fingernails and toenails) were seen more than twice as often in the closed group than in the family ward patients ( 38 versus 18 ), which can be due to a lack of self-care, repeated trauma, self-inflicted trauma, and possibly being on a fixed diet for years, leading to vitamin and mineral deficiencies in closed ward patients. Ridging was the most common finding in both groups. Median canalicular dystrophy was seen in six patients in the closed group and none in the family group, which was possibly due to repeated and self-inflicted trauma. Ten patients had dystrophic nails in the closed group and only two in the family group, which was ascribed to longstanding fungal infections and repeated trauma. Pitting was seen in three patients in the family ward (two of whom had psoriasis and one with alopecia areata) and none in the closed ward. Nail plate thickening, petechial hemorrhage, and traumatic nails were seen more in closed ward patients. One patient in the family ward had unidigital clubbing, which was due to repeated trauma.

\section{Conclusion}

Skin manifestations are more common in chronic neglected psychiatric patients under custodial care, which can be explained due to their chronicity of disease, lack of self- and family care, and less attention and care provided by healthcare workers. Moreover, distinct differences were observed between the family ward setting and closed ward patients regarding the pattern of dermatoses. The authors therefore stress upon the importance of familial care to be provided to psychiatric patients living in custodial settings, which will certainly affect their skin care.

\section{References}

1. Jafferany M. Psychodermatology: a guide to understanding common psychocutaneous disorders. Prim Care Companion J Clin Psychiatry. 2007;9:203-13.

2. Humphreys F, Humphreys MS. Psychiatric morbidity and skin disease: what dermatologists think they see. Br J Dermatol. 1998;139:679-81.

3. Attah JFY, Mostaghimi H. Co-morbidity between dermatological diseases and psychiatric disorders in Papua New Guinea. Int J Dermatol. 1995;34:244-8.

4. Folks DG, Warnock JK. Psychocutaneous disorders. Curr Psychiatry Rep. 2001;3:219-25.

5. Amirnia M, Ganji S, Arfaei A, Khanmohammmadi M. Survey of skin disease in schizophrenia patients visiting psychiatry clinics in Razi and Sina hospital in 2007 to 2008, Tabriz in Iran. Ann Biol Res. 2011;2:120-4.

6. American Psychiatric Association. Diagnostic and statistical manual of mental disorders. 4th ed. Washington, DC; 1994.
7. Mookhoek EJ, van de Kerkhof PCM, Hovens JEJM, Brouwers JRBJ, Loonen AJM. Substantial skin disorders in psychiatric illness coincide with diabetes and addiction. JEADV. 2011;25:392-7.

8. Kuruvila M, Gahalaut P, Zacharia A. A study of skin disorders in patients with primary psychiatric conditions. Indian J Dermatol Venereol Leprol. 2004;70:292-5.

9. Margoob MA, Dutta KS. Drug abuse in Kashmir-experience from a psychiatric disease hospital. Indian J Psychiat. 1993;35:163-5.

10. de Jong JT, Komproe IH, van Ommeren M, El Masri M, Araya M, Khaled N, et al. Lifetime events and posttraumatic stress disorder in 4 post conflicts settings, JAMA. 2001;86:555-62.

11. Rather YH, Bashir W, Sheikh AA, Amin M, Zahgeer YA. Socio-demographic and clinical profile of substance abusers attending a regional drug de-addiction centre in chronic conflict area: Kashmir, India. Malays J Med Sci. 2013;20:31-8. 
12. Shaffer D, Gould M, Fisher P, Trautman P, Moreau D, Kleinman M. Psychiatric diagnosis in child and adolescent suicide. Arch Gen Psychiatry. 1996;53:339-48.

13. Williams K. Tattoos, scars, body adornment and dishevelment in an acute psychiatric population. Psychiatr Bull. 1998;22:94-6.

14. Rhodes AR. Public education and cancer of the skin. What do people need to know about melanoma and nonmelanoma skin cancer? Cancer. 1995;75:613-36.

15. Hassan I, Sajad P, Reshi R. Histopathological analysis of the cutaneous changes due to kangri use in Kashmiri population: a hospital based study. Indian J Dermatol. 2013;58:188-90.

16. Sarkany RPE, Breathnach SM, Morris AAM, Weismann K, Flynn PD. Metabolic and nutritional disorders. In: Burns T, Breathnach S, Cox N, Griffiths C, editors. 8th ed. Rook's textbook of dermatology. London: Wiley-Blackwell; 2010. p. 3:59.44-47.
17. Mahendiran S, Burkhart CN, Burkhart CG. Hyperhidrosis: a review of a medical condition. Burkhart. Open Dermatol J. 2009;3:195-7.

18. Millard LG, Millard J. Psychocutaneous disorders. In: Burns T, Breathnach S, Cox N, Griffiths C, editors. Rook's textbook of dermatology. 8th ed. London: WileyBlackwell; 2010. p. 4:64.13-16.

19. Lyell A. Delusions of parasitosis. Br J Dermatol. 1983;108:485-99.

20. Millard LG, Millard J. Psychocutaneous disorders. In: Burns T, Breathnach S, Cox N, Griffiths C, editors. Rook's textbook of dermatology. 8th ed. London: WileyBlackwell; 2010. p. 4:64.30-33. 